\title{
Ruxolitinib for steroid-refractory acute graft-versus-host disease: case series and literature review
}

\author{
Adrianna Spałek, Krzysztof Woźniczka, Anna Armatys, Agata Wieczorkiewicz-Kabut, \\ Izabela Noster, Grzegorz Helbig* iD \\ Department of Hematology and Bone Marrow Transplantation, School of Medicine in Katowice, Medical University of Silesia, \\ Katowice, Poland
}

\section{Introduction}

Acute graft-versus-host-disease (aGvHD) is one of the leading causes of mortality after allogeneic hematopoietic stem cell transplantation (allo-HSCT). Despite the availability of different strategies of prophylaxis, $15 \%$ of patients suffer from life threatening grade III-IV aGVHD with severe liver impairment and/or massive diarrhea [1].

Fifty years after the worldwide introduction of allo-HSCT, the use of methylprednisolone (MP) remains the only accepted first-line treatment for aGvHD. Unfortunately, MP is not effective in almost $60 \%$ of patients with grade IV disease [2]. Steroid-refractory aGvHD (SR-aGvHD) is associated with a poor outcome, with only a $5-30 \%$ survival rate [1]. Despite much work in this field, second-line treatment for SR-aGVHD has yet to be established, and much depends on the experience of the transplant center.

In May 2019, the US Food and Drug Administration (FDA) approved ruxolitinib (RUX), a JAK2 inhibitor for the treatment of SR-aGvHD in adult and pediatric patients aged 12 and above [3]. Approval was based on the results of the REACH 2 clinical trial in which therapy of SR-aGvHD with RUX led to significant improvements in outcomes compared to other immunosuppressive therapies [4].

We describe below our experience with RUX in therapy of gut and liver SR-aGvHD through two descriptive cases.

\section{Material and methods}

We conducted a selective review of allo-HSCT recipients who received RUX for SR-aGvHD in 2020. All study patients provided written informed consent for therapy.

\section{Results and discussion}

\section{Patient 1}

A 37-year-old male diagnosed with myelodysplastic syndrome with multilineage dysplasia underwent allo-HSCT from a 10/10 human leukocyte antigen (HLA)-matched unrelated donor in August 2020.

His conditioning regimen included busulfan and cyclophosphamide (BuCy). GvHD prophylaxis consisted of cyclosporine (CsA), methotrexate (Mtx) and rabbit ATG (thymoglobulin). The patient achieved neutrophil and platelet engraftments on days +15 and +13 respectively. The early aplastic post-transplantation period was complicated by fever of unknown origin, with an increase in C-reactive protein (CRP) level up to $274 \mathrm{mg} / \mathrm{L}$ (normal range $<5 \mathrm{mg} / \mathrm{L}$ ). On day +14 , he developed an erythematous maculopapular rash on $>50 \%$ of his body surface (grade $\mathrm{II}$ aGvHD) and required pulses of MP at $1 \mathrm{mg} / \mathrm{kg}$ for a couple of days, with rapid resolution. Due to increased serum creatinine level, CsA was switched to mycophenolate mofetil (MMF).

Several days later, mild watery diarrhea (less than $500 \mathrm{~mL} /$ day) appeared. Clostridioides difficile infection and other possible causes of diarrhea were carefully excluded. $\mathrm{MP}$ at $2 \mathrm{mg} / \mathrm{kg} /$ day and oral budesonide were administered due to rapid progression of intestinal symptoms $(>1,500 \mathrm{~mL}$ of watery stool a day and worsening abdominal pain). The patient was diagnosed with intestinal grade III aGvHD [5]. Five days later, the patient's condition deteriorated and subileus developed. MP was gradually tapered, and tacrolimus (TAC) in continuous infusion was attempted. Two days later, TAC was stopped due to repeated episodes of acute renal failure. RUX was started at $5 \mathrm{mg}$ twice daily from day +28 . Stool volume steadily decreased to less than $200 \mathrm{ml}$ on

\footnotetext{
*Address for correspondence: Grzegorz Helbig, Department of Hematology and Bone Marrow Transplantation, Medical University of Silesia, Dąbrowskiego 25, 40-032 Katowice, Poland, phone +48 3225913 10, fax +48 3225549 85, e-mail: ghelbig@o2.pl Received: 7.09.2021 Accepted: 10.10.2021
} 
day +32 and abdominal cramps significantly diminished. A repeated bone marrow aspiration biopsy performed on day +28 revealed complete remission with full donor chimerism. On day +47 , the patient was discharged on maintained doses of oral MP, MMF and RUX ( $5 \mathrm{mg}$ twice daily).

On day +56 , the patient was urgently readmitted to hospital because of progressive pancytopenia probably due to the myelosuppressive effect of RUX. Bone marrow biopsy on day +60 showed normocellular marrow with normal myeloid maturation. Full donor chimerism was documented on reverse-transcriptase polymerase chain reaction (RT-PCR). Cytomegalovirus (CMV) reactivation was excluded. Due to severe thrombocytopenia, MP at $32 \mathrm{mg}$ daily was given. RUX was reduced to $5 \mathrm{mg}$ daily while MMF was maintained. Unfortunately, the patient died several weeks later due to massive bleeding from the upper gastrointestinal tract as a consequence of thrombocytopenia.

\section{Patient 2}

A 32-year-old female with myeloid blast crisis of chronic myeloid leukemia $(\mathrm{CML})$ received stem cells from a brother in December 2019. The procedure was performed in active disease after ineffective salvage regimen. Thiotepa, busulfan and fludarabine (TBF) were administered as conditioning. GvHD prophylaxis consisted of CsA and Mtx. An early aplastic phase was uncomplicated. Neutrophil and platelet engraftments occurred on days 12 and 11, respectively. Bone marrow assessment on day +29 revealed complete hematological remission with full donor chimerism. The BCR-ABL(p210) transcript was undetectable. Due to the absence of aGvHD symptoms and the high risk of disease recurrence, donor lymphocyte infusion (DLI) of $1 \times 10^{7} \mathrm{CD} 3$ positive cells was administered on day +29 . The patient was discharged on tapering doses of CsA.

On day +133 while free of any immunosuppressive therapy, she was urgently readmitted to hospital because of jaundice and dark-coloured urine. Bilirubin concentration was significantly increased to $190 \mu \mathrm{mol} / \mathrm{L}$ (normal range 5-21 $\mu \mathrm{mol} / \mathrm{L}$ ) with marked elevation of alanine aminotransferase (346 IU/L; normal range <35 IU/L), alkaline phosphatase (169 IU/L; normal range 30-120 IU/L) and gamma-glutamyl transferase (701 IU/L; normal range <38 IU/L). Cholestasis as well as hepatitis $B$ and $C$ reactivations were excluded. The patient was diagnosed with hepatic grade III aGvHD. Skin and gut were free of aGVHD. The patient received MP at $1 \mathrm{mg} / \mathrm{kg}$ intravenously for 13 days with a subsequent slow reduction and conversion to oral therapy. Serum bilirubin concentration diminished to $97 \mu \mathrm{mol} / \mathrm{L}$ and remained stable. RUX at $5 \mathrm{mg}$ twice daily was added to oral MP and bilirubin concentration diminished to $37 \mu \mathrm{mol} / \mathrm{L}$. The dose of RUX was reduced to $7.5 \mathrm{mg}$ per day. Currently, two years after transplantation, the patient remains free of immunosuppressive treatment with no symptoms of GvHD. There was no reactivation of CMV during RUX treatment.
The treatment of SR-aGvHD has remained unsatisfactory for decades. Patients with SR aGVHD are generally treated with different immunosuppressive agents with variable effects [1]. RUX by selective inhibition of JAK1 and JAK2 has been proven to block the action of proinflammatory cytokines signaling through the JAK-STAT pathway such as interleukin-1 and -6, tumor necrosis factor, and interferon-gamma [6]. This results in reduced proliferation of effector T-cells and an increase of regulatory T-cells in the colon and liver, leading to the attenuation of aGvHD symptoms [7].

In the pivotal REACH-1 trial, the administration of RUX in SR aGvHD patients resulted in a nearly $55 \%$ overall response rate, including $27 \%$ with complete responses at day 28. RUX treatment started from $5 \mathrm{mg}$ twice daily, and the dose was escalated to $10 \mathrm{mg}$ twice daily when no toxicity was present. The highest response rate was observed in skin (61\%), then in the upper (45\%), and the lower (46\%) gastrointestinal tract, and the liver (26\%) [8]. An even higher overall response rate (70\%) was demonstrated in a study by a Spanish group. The response to RUX was achieved after a median of two weeks of treatment with overall survival for responders of $62 \%$ vs. $28 \%$ for non-responders [9]. Although RUX has a favourable toxicity profile, the most common side effects concern cytopenias and infectious complications $[4,10]$. Anemia and thrombocytopenia were seen in $65 \%$ and $62 \%$ of patients in the REACH-1 trial, respectively [8]. In another study, of RUX-treated patients, $68 \%$ had at least one episode of infection complication. Among infections, CMV and human herpesvirus 6 (HHV-6) viremia were demonstrated in $21 \%$ and $11 \%$ of patients respectively. Bacteremias were recorded in $42 \%$ of patients, whereas no fungal infection was noted [10].

Although intensive immunosuppression reduces the beneficial graft-versus-leukemia effect and may lead to potential recurrence of prior malignancy [11], therapy with RUX has not been associated with higher frequency of hematological relapse [12]. The relapse rate during RUX for SR aGVHD varies between $1.2 \%$ and $9.3 \%$ depending on the published data $[9,13]$.

With the increasing use of RUX, a definition of RUX refractoriness should be established. Progression of GvHD after at least 5-10 days of RUX, a lack of improvement in GvHD symptoms $\geq 14$ days of RUX, or a worsening of GvHD after initial improvement, define RUX-refractory aGvHD and necessitate alternative treatment [14].

RUX has not yet been reimbursed for Polish patients with GvHD, and the drug in our cases was obtained through the Managed Access Program of the Novartis company. Our clinical experience is limited to fewer than 15 cases (herewith we have presented two illustrative patients) treated exclusively for hepatic and intestinal manifestations of SR aGvHD. We have never used RUX for patients with SR cutaneous aGvHD, and therefore we cannot judge its efficacy in this patient population. 
According to our observations, RUX treatment seems to be more effective in liver involvement than in intestinal, although this is in contrast to the results of the REACH-1 trial where the lowest efficacy was demonstrated for liver manifestation [8]. The latter was also true in a recently published study of patients after haploidentical transplantation [15]. However, more data is needed on particular organ response. Regarding our patient population, the starting dose of RUX varied from $5 \mathrm{mg}$ daily to $5 \mathrm{mg}$ twice daily, and none of the patients received $10 \mathrm{mg}$ twice daily. Most patients, if not all, developed or worsened pancytopenia as a consequence of RUX therapy, other co-used therapies and concomitant bacterial or viral infections.

Based on our limited experience, $10 \mathrm{mg}$ daily of RUX should not be exceeded. Due to the difficult access to RUX in Poland, it is usual to only administer it as a third or further line of therapy when other immunosuppressive agents have failed to achieve a response and the patient remains in a serious condition with concomitant pancytopenia associated with infection. Thus, it seems reasonable to administer RUX at an early stage of SR aGvHD treatment, but this requires a system solution allowing easy access to the drug.

In conclusion, RUX represents a valuable therapeutic option for patients with SR-aGvHD. Our experience is limited to case series. Easier access to RUX leading to its broader use would be required to obtain more conclusive data.

\section{Authors' contributions}

AS, GH - planned study, wrote manuscript, analyzed data; KW, AA, AWK, IN - collected data, critical review.

\section{Conflictsof interest}

GH - advisory board Novartis, Abbvie, speaker fee Novartis; AS - speaker fee Novartis; KW, AA, AWK, IN - none.

\section{Financial suport}

None.

\section{Informed consent}

Informed consent was obtained from all patients participating in this study.

\section{Ethics}

All procedures performed in studies involving human participants were in accordance with the ethical standards of the institutional and/or national research committee and with the 1964 Helsinki Declaration and its later amendments or comparable ethical standards.

\section{References}

1. Martin PJ, Rizzo JD, Wingard JR, et al. First- and second-line systemic treatment of acute graft-versus-host disease: recommendations of the American Society of Blood and Marrow Transplantation. Biol
Blood Marrow Transplant. 2012; 18(8): 1150-1163, doi: 10.1016/j. bbmt.2012.04.005, indexed in Pubmed: 22510384.

2. Ruutu T, Gratwohl A, de Witte T, et al. Prophylaxis and treatment of GVHD: EBMT-ELN working group recommendations for a standardized practice. Bone Marrow Transplant. 2014; 49(2): 168-173, doi: 10.1038/bmt.2013.107, indexed in Pubmed: 23892326.

3. Przepiorka D, Luo L, Subramaniam S, et al. FDA approval summary: ruxolitinib for treatment of steroid-refractory acute graft-versus-host disease. Oncologist. 2020; 25(2): e328-e334, doi: 10.1634/theoncologist.2019-0627, indexed in Pubmed: 32043777.

4. Zeiser R, von Bubnoff N, Butler J, et al. REACH2 Trial Group. Ruxolitinib for glucocorticoid-refractory acute graft-versus-host disease. N Engl J Med. 2020; 382(19): 1800-1810, doi: 10.1056/NEJMoa1917635, indexed in Pubmed: 32320566.

5. Przepiorka D, Weisdorf D, Martin P, et al. 1994 Consensus Conference on Acute GVHD Grading. Bone Marrow Transplant. 1995; 15(6): 825-828, indexed in Pubmed: 7581076.

6. Elli EM, Baratè $\mathrm{C}$, Mendicino $\mathrm{F}$, et al. Mechanisms underlying the anti-inflammatory and immunosuppressive activity of ruxolitinib. Front Oncol. 2019; 9: 1186, doi: 10.3389/fonc.2019.01186, indexed in Pubmed: 31788449.

7. Spoerl S, Mathew NR, Bscheider M, et al. Activity of therapeutic JAK 1/2 blockade in graft-versus-host disease. Blood. 2014; 123(24): 3832-3842, doi: 10.1182/blood-2013-12-543736, indexed in Pubmed: 24711661.

8. Jagasia M, Perales MA, Schroeder M, et al. Results from REACH1, a single-arm phase 2 study of ruxolitinib in combination with corticosteroids for the treatment of steroid-refractory acute graft-vs-host disease. Blood. 2018; 132(Suppl 1): 601-601, doi: 10.1182/ blood-2018-99-116342.

9. Gomez VE, Garcia-Gutierrez V, Corral LL, et al. Ruxolitinib in refractory acute and chronic graft-versus-host disease: a multicenter study. Bone Marrow Transplant. 2020; 55(3): 641-648, doi: 10.1038/ s41409-019-0731-x, indexed in Pubmed: 31700138.

10. Abedin S, McKenna E, Chhabra S, et al. Efficacy, toxicity, and infectious complications in ruxolitinib-treated patients with corticosteroid-refractory graft-versus-host disease after hematopoietic cell transplantation. Biol Blood Marrow Transplant. 2019; 25(8): 1689-1694, doi: 10.1016/j.bbmt.2019.04.003, indexed in Pubmed: 30965140.

11. Chang YJ, Zhao XY, Huang XJ. Strategies for enhancing and preserving anti-leukemia effects without aggravating gaft-versus-host disease. Front Immunol. 2018; 9: 3041, doi: 10.3389/fimmu.2018.03041, indexed in Pubmed: 30619371.

12. Choi J, Cooper ML, Alahmari B, et al. Pharmacologic blockade of JAK1/JAK2 reduces GvHD and preserves the graft-versus-leukemia effect. PLoS One. 2014; 9(10): e109799, doi: 10.1371/journal. pone.0109799, indexed in Pubmed: 25289677.

13. Zeiser R, Burchert A, Lengerke C, et al. Ruxolitinib in corticosteroid-refractory graft-versus-host disease after allogeneic stem cell transplantation: a multicenter survey. Leukemia. 2015; 29(10): 2062-2068, doi: 10.1038/leu.2015.212.

14. Mohty M, Holler E, Jagasia M, et al. Refractory acute graft-versus-host disease: a new working definition beyond corticosteroid refractoriness. Blood. 2020; 136(17): 1903-1906, doi: 10.1182/ blood.2020007336, indexed in Pubmed: 32756949.

15. Liu Y, Fan Yi, Zhang W, et al. Efficiency and toxicity of ruxolitinib as the salvage treatment in steroid-refractoryc acute graft-versus-host disease after haplo-identical stem cell transplantation. Transplant Cell Ther. 2021; 27(4): 332.e1-332.e8, doi: 10.1016/j.jtct.2021.01.019, indexed in Pubmed: 33836880. 